\title{
The role of specific interactions on dynamical processes in a room temperature ionic liquid
}

\author{
K S MALI \\ Radiation and Photochemistry Division, Bhabha Atomic Research Centre, Trombay, Mumbai 400085 \\ e-mail: ksmali81@gmail.com
}

MS received 19 June 2008; revised 16 September 2008

\begin{abstract}
This article describes our ongoing efforts to comprehend the role of specific interactions on the dynamical processes such as rotational diffusion and photoisomerization in a typical room temperature ionic liquid. Rotational diffusion studies carried out with a pair of structurally similar non-dipolar solutes indicate that organic solutes do experience strong specific interactions even with the highly associative ionic liquids such that their rotation is hindered. Similar measurements carried out with a nonpolar and a dipolar solute in an ionic liquid and a conventional solvent reveal that even in ionic liquids, apart from the viscosity of the medium, the important parameters, which govern the solute rotation are the solvent-size and free volume in case of non-polar solutes, whereas for charged and dipolar solutes, it is the solute-solvent interaction strength. Photoisomerization studies dealing with a pair of carbocyanine derivatives have shed light on the influence of solvent viscosity and specific interactions on the rates of photoisomerization. Our results point to the fact that the positively charged as well as the negatively charged cyanine derivatives do not experience specific interactions with the ionic liquid such that the isomerization rates are affected. However, when the isomerization rates are compared with a conventional isoviscous solvent, it has been noticed that the rates of isomerization are solely governed by viscosity of the medium in case of the positively charged cyanine derivative. In contrast, photoisomerization rates of the negatively charged cyanine derivative are significantly faster in a conventional isoviscous solvent compared to the ionic liquid due to the specific interactions between the solute and the former, which lower the barrier height for isomerization.
\end{abstract}

Keywords. Ionic liquid; rotational diffusion; photoisomerization; specific interactions.

\section{Introduction}

Most chemical reactions to date have been carried out in molecular solvents. Thus, our understanding of chemical reactions is largely based on the behaviour of reactants in the conventional molecular solvents. However, this scenario has begun to change with the emergence of a new class of solvents known as ionic liquids. Ionic liquids are a special class of molten salts. There are numerous ways in which an ionic liquid can be defined, and perhaps Welton's definition is the most widely accepted. ${ }^{1}$

\subsection{A material that is entirely composed of ions, and has a melting point below $373 \mathrm{~K}$}

Besides this definition, ionic liquids are also sometimes referred to as molten salts, non-aqueous ionic liquids or room temperature ionic liquids, and all these terms are considered to be entirely valid.
These liquids have attracted considerable interest in recent times because of their utility as 'green' alternatives to volatile organic solvents for carrying out synthesis and catalysis. ${ }^{1-7}$ However, the 'green' facet (environmentally benign nature) of these liquids is still open to debate ${ }^{8,9}$ as the chemicals used for their synthesis are not necessarily green. Because of their negligible vapor pressure and high liquidus range, ionic liquids are also being considered as the "missing link' between conventional solvents, which can be handled up to $373 \mathrm{~K}$ and classical molten salts with melting points greater than $673 \mathrm{~K} .{ }^{10}$

In addition to an increased attention of researchers from diverse disciplines, ionic liquids are enjoying a plethora of applications in sensors, solar cells, solid state photocells, batteries, lubricants, etc. ${ }^{6,7}$ Despite numerous applications in various domains of science, the fundamental physical properties of these liquids are still poorly understood. In order to exploit the tremendous potential of these fascinating 
liquids, it is imperative to have a thorough grasp on their physical properties, especially the nature of interactions between the cations and anions that constitute the ionic liquids and their interactions with the added solutes as well. Although, a significant progress has been made towards the understanding of solute-solvent interactions in conventional molecular solvents, this aspect is still in its infancy in ionic liquids. Nevertheless, it has been well-established that ionic liquids can be viewed as threedimensional network of cations and anions held together by interactions such as Coulombic forces, hydrogen bonds and dispersive forces. ${ }^{3,11}$ Due to these strong interactions between the constituent cations and anions, ionic liquids have high viscosities. ${ }^{2,12-14}$ However, compared to the amount of literature published on preparation and possible applications of ionic liquids, little is known about the dynamics of solute molecules solubilized in these liquids. Only a limited number of reports are available in the literature that deal with dynamics of solute molecules solubilized in these novel media as evident from the brief overview given below.

In recent times, rotational diffusion of organic solutes has been examined in ionic liquids to find out whether it is any different from that observed in conventional solvents. ${ }^{14-19}$ The findings of these investigations reveal that solute rotation is sluggish compared to that in conventional solvents and the observed slow reorientation times have been ascribed to the high viscosity of the ionic liquids. Baker et $a l^{14}$ have measured the reorientation times of a large non-polar solute BTBP (N,N-bis(2,5-di-tert-butylphenyl)-3,4,9,10-perylenedicaboximide) in ionic liquid 1-butyl-3-methylimidazolium hexafluorophosphate $\left(\left[\mathrm{bmim}^{+}\right]\left[\mathrm{PF}_{6}^{-}\right]\right)$as a function of temperature and concluded that a single layer of $\left[\mathrm{bmim}^{+}\right]$or $\left[\mathrm{PF}_{6}^{-}\right.$ ] associates with the solute. However, analysis of the same data by Ingram et $a l^{15}$ has revealed that the conclusion reached by Baker et $a l^{14}$ is a consequence of modelling BTBP as a sphere instead of an ellipsoid. Ingram et $a l^{15}$ have also measured the reorientation times of the solute 4-AP (4-aminophthalimide) in $\left[\mathrm{bmim}^{+}\right]\left[\mathrm{PF}_{6}^{-}\right]$and other conventional solvents and found that its rotational behaviour in ionic liquid is similar to that expected in a viscous solvent. In addition to these investigations, Ito et $a l^{17}$ have examined the rotational diffusion of a number of neutral and charged solutes in the above-mentioned ionic liquid and concluded that even for ionic solutes; chargecharge component of the solute-solvent interaction is not of primary importance for determining rota- tional friction. The most probable reason for the observed behaviour is the strong association between the cation and the anion of the ionic liquid, which leads to the formation of ion pairs or even higher aggregates. ${ }^{11,20}$ Thus, the highly associative nature of the ionic liquid prevents it from experiencing strong specific interactions with the solute molecules. Whether this aspect is universal or specific to a particular solute has not been examined so far.

Apart from rotational diffusion studies, solvation dynamics investigations have also been carried out in different classes of ionic liquids to understand the reorganization of the solvent around a solvatochromic probe after a sudden change of the probe's dipole moment through optical excitation of the probe. ${ }^{21-27}$ These studies are essential to comprehend solventcontrolled chemical reactions. The solvation dynamics in ionic liquids has been found to be biphasic consisting of a short and a long component. Despite a vast number of studies, no clear-cut mechanism of the solvation process in ionic liquids could be established. ${ }^{26}$ However, recent measurements dealing with the complete solvation response in ionic liquids indicate that the solvation in imidazolium and pyrrolidium liquids is biphasic consisting of a minor sub-picosecond component associated with the inertial solvent motions and a broadly distributed slow component coupled to the structural reorganization of the solvent. ${ }^{27}$ Besides these investigations, a very recent study indicates that the solvation dynamics measurements in ionic liquids are akin to those in conventional polar solvents in the sense that both the measurements effectively measure the dielectric response of these systems. Furthermore, it also demonstrates that, if proper molecular correlations are taken into account, theories that use dielectric relaxation data can be semi-quantitatively applied to explain the existing experimental results. ${ }^{28}$

The above-mentioned processes have also been investigated in composite systems in which ionic liquid is one of the components. Such systems include mixtures of ionic liquid with conventional polar and non-polar solvents and also organized assemblies, which comprise of an ionic liquid. Rotational diffusion and solvation dynamics has been investigated in mixtures of ionic liquid with polar solvents such as water, methanol and acetonitrile. ${ }^{18,19,29}$ These studies reveal that solvation as well as rotational dynamics becomes faster with an increase in the amount of polar solvent which has been attributed to decrease in the viscosity of the ionic liquid. Addition of non-polar solvents such as toluene and 1,4- 
dioxane has been found to produce a similar effect on the dynamics. Solvation as well as rotational relaxation times decrease with an increase in the amount of non-polar solvent. ${ }^{30}$

Recent studies carried out with anionic and nonionic surfactants indicate that these systems aggregate to form micelles and reverse micelles in ionic liquids analogous to water. ${ }^{31-38} \mathrm{~A}$ few reports are available in literature that deal with solvation and rotational dynamics of hydrophobic probes in these systems. ${ }^{39-42}$ It has been observed that solvation as well as rotational dynamics of the probe coumarin 153 is slower in Brij-35 micelles formed in $\left[\mathrm{bmim}^{+}\right]$ $\left[\mathrm{PF}_{6}^{-}\right]$compared to that observed in neat $\left[\mathrm{bmim}^{+}\right]$ $\left[\mathrm{PF}_{6}^{-}\right]{ }^{39}$ Similar studies ${ }^{40}$ have also been carried out in micelles formed with poly(oxyethylene glycol)ethers in 1-butyl-3-methylimidazolium tertafluoroborate $\left(\left[\mathrm{bmim}^{+}\right]\left[\mathrm{BF}_{4}^{-}\right]\right)$. It has been observed that the solvation times of $\mathrm{C} 153$ are longer in these micelles compared to neat $\left[\mathrm{bmim}^{+}\right]\left[\mathrm{BF}_{4}^{-}\right]$. However, the rotational relaxation times are longer in the micelles compared to neat $\left[\mathrm{bmim}^{+}\right]\left[\mathrm{BF}_{4}^{-}\right] .^{40}$ Besides these studies, solvation and rotational dynamics has also been investigated in Triton X-100/cyclohexane microemulsions with the ionic liquid $\left[\mathrm{bmim}^{+}\right]\left[\mathrm{BF}_{4}^{-}\right]$as the core. ${ }^{41}$ According to this study, there is an enhancement in the average reorientation time of the probe with an increase in the mole ratio of the ionic liquid to the surfactant, which has been attributed to the accumulation of highly viscous $\left[\mathrm{bmim}^{+}\right]\left[\mathrm{BF}_{4}^{-}\right]$in the core of the microemulsion. ${ }^{41}$ Similar study has also been carried out in $\left[\mathrm{bmim}^{+}\right]\left[\mathrm{PF}_{6}^{-}\right] /$Triton $\mathrm{X}-100 /$ water ternary system. ${ }^{42}$ The outcome of this investigation indicates that for hydrophobic solute C153, the solvation and rotational relaxation times remain unchanged with an increase in the mole ratio of the ionic liquid to the surfactant. This has been ascribed to the location of $\mathrm{C} 153$ in the microemulsion, which remains unchanged with an increase in mole ratio of the ionic liquid to the surfactant. On the other hand, in case of $\mathrm{C} 151$, which is a hydrophilic probe, the fast component of the solvation time gradually increases and the slow component decreases. The rotational relaxation time has been found to increase with an increase in mole ratio of the ionic liquid to the surfactant, which has been attributed to an increase in the number of $\mathrm{C} 151$ molecules in the core of the microemulsion. ${ }^{42}$

Photoisomerization studies in ionic liquids, on the other hand, are even sparse and such studies have begun only recently. ${ }^{29,43-45}$ In view of the prevailing situation, our efforts have been directed towards in- vestigating rotational diffusion ${ }^{46,47}$ and photoisomerization ${ }^{48}$ in a typical room temperature ionic liquid, $\left[\mathrm{bmim}^{+}\right]\left[\mathrm{PF}_{6}^{-}\right]$. This ionic liquid has been chosen because it is amongst the widely used ionic liquids whose physical properties have been well characterized. Since the above-mentioned dynamical processes depend on the frictional coupling between the solute and the solvent, these investigations have been undertaken to address the following issues: (i) Do organic solutes experience specific interactions with ionic liquids? (ii) Do ionic interactions hinder the rotational diffusion of charged solutes in an ionic liquid? (iii) What governs the rotational diffusion of a solute in absence of specific interactions? (iv) What is the role of solvent viscosity and specific solute-solvent interactions on the rates of photoisomerization in an ionic liquid? This short review will provide answers to these questions by illustrating examples from our recent work that has been carried out in $\left[\mathrm{bmim}^{+}\right]\left[\mathrm{PF}_{6}^{-}\right]$with the aid of steady-state and time-resolved fluorescence spectroscopy.

\section{Rotational diffusion studies}

Rotational diffusion studies of small and mediumsized solute molecules dissolved in liquids have been extensively employed to explore solute-solvent interactions. ${ }^{49-52}$ In such studies, the experimentally measured reorientation time of a solute molecule is compared to the one calculated using the diffusionbased hydrodynamic theory of Stokes-Einstein-Debye (SED) with slip or stick boundary conditions. ${ }^{49-53}$ Though originally intended to describe rotational diffusion of macroscopic objects, the SED theory has met with a reasonable degree of success even at the molecular level. However, deviations from the predictions of SED theory have often been observed because it does not take into consideration the size of the solvent molecule with respect to the size of the rotating solute, chemical nature of the solute and the solvent and also the non-specific interactions arising due to the dielectric properties of the solute and the solvent known as 'dielectric friction'.

\subsection{Do organic solutes experience specific interactions with ionic liquids?}

In an attempt to find out whether organic solutes experience specific interactions with the highly associative ionic liquids, rotational diffusion of two structurally similar non-dipolar solutes; 2,5-dimethyl-1,4-dioxo- 
3,6-diphenylpyrrolo[3,4-c]pyrrole (DMDPP) and 1,4-dioxo-3,6-diphenylpyrrolo[3,4-c]pyrrole (DPP) has been investigated in $\left[\mathrm{bmim}^{+}\right]\left[\mathrm{PF}_{6}^{-}\right]$as a function of temperature. Figure 1 gives the molecular structures of DMDPP and DPP. It is evident from the figure that the two solutes are not only structurally similar but are also comparable in size and the sole difference between them is the presence of secondary amino groups in case of DPP. Because of the presence of these secondary amino groups, DPP interacts strongly with a number of solvents and as a consequence rotates significantly slower compared to DMDPP. On the other hand, the interactions between the two carbonyl groups of DMDPP and a majority of solvents are not strong enough to hinder
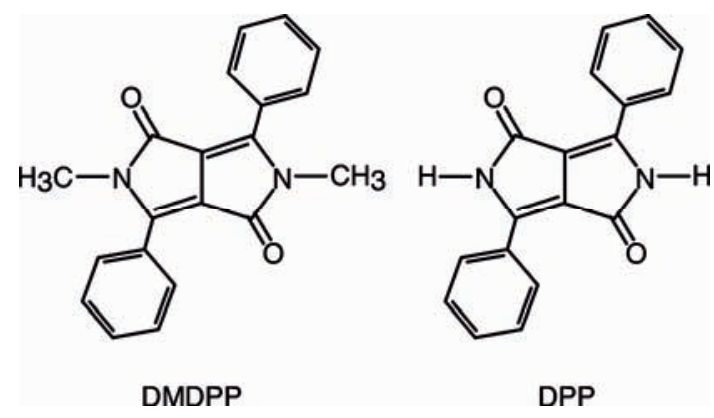

Figure 1. Molecular structures of the solutes DMDPP and DPP

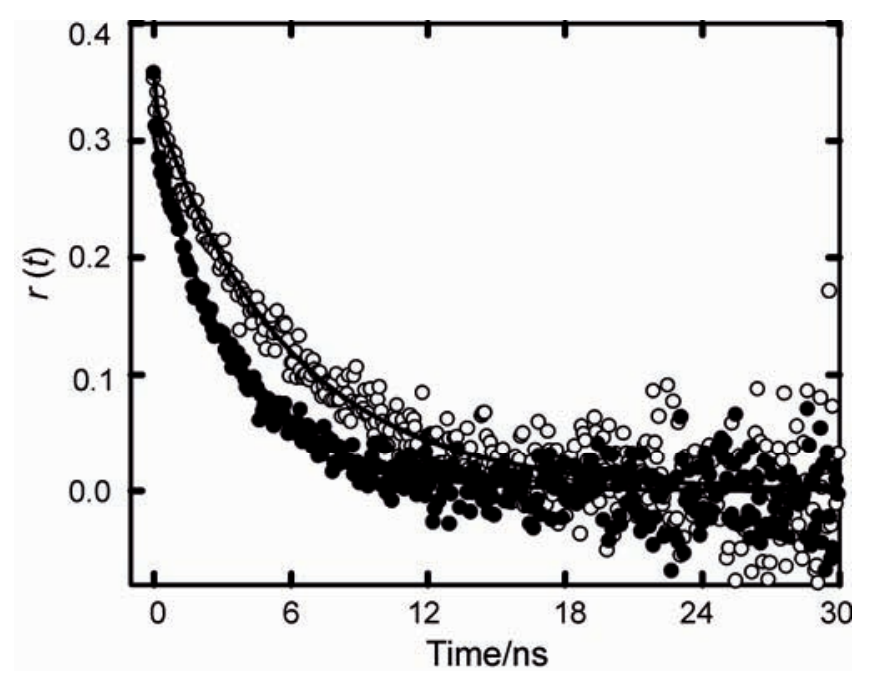

Figure 2. Anisotropy decays of DMDPP (•) and DPP (O) in $\left[\mathrm{bmim}^{+}\right]\left[\mathrm{PF}_{6}^{-}\right]$at $313 \mathrm{~K}$. The smooth lines passing through the experimental data points are the fitted ones. Reprinted with permission from ref. 46. Copyright (2005) American Institute of Physics. its rotation. Hence, DMDPP experiences only hydrodynamic or mechanical friction in these solvents. However, in case of strong hydrogen bond donating solvents such as trifluoroethanol, the rotation of DMDPP is also influenced due to specific interactions. ${ }^{54}$ Moreover, it has been observed that the reorientation times of DPP have been found to depend on the strength of the interaction between solute and the solvent. The rotational diffusion of DMDPP and DPP has been extensively investigated in conventional solvents and the important findings have been summarized. ${ }^{52}$ Considering the fact that DPP is a strong hydrogen bond donor, it is expected to experience strong specific interactions with the anion $\left[\mathrm{BF}_{6}^{-}\right]$of the ionic liquid. Nevertheless, it remains to be seen whether the ionic liquid can experience specific interactions with the added solute DPP by overcoming the cohesive forces that are present between the constituent cations and anions.

To this effect, rotational diffusion of DMDPP and DPP has been investigated in $\left[\mathrm{bmim}^{+}\right]\left[\mathrm{PF}_{6}^{-}\right]$over the temperature range $298-363 \mathrm{~K}$ by measuring the fluorescence anisotropy decays. Figure 2 displays typical anisotropy decay curves of the two solutes at $313 \mathrm{~K}$ and it is evident from the figure that the rotation of DPP is significantly slower compared to that of DMDPP. In order to understand the rotational diffusion of DMDPP and DPP in $\left[\mathrm{bmim}^{+}\right]\left[\mathrm{BF}_{6}^{-}\right]$, the SED theory ${ }^{49-53}$ has been used as the primary tool. According to the SED theory, the reorientation time $\tau_{r}$ of a solute molecule is proportional to its size or hydrodynamic volume and viscosity of the solvent at a particular temperature and is given by

$$
\tau_{r}=\frac{\eta V}{k T}(f C)
$$

where $k$ and $T$ are the Boltzmann constant and absolute temperature, respectively. $\eta$ is the viscosity of the solvent and $V$ is the van der Waals volume of the solute. The parameter $f$ is the shape factor introduced to account for the shapes of the solute molecules, which are often non-spherical. ${ }^{55}$ The shapes of the solute molecules are usually incorporated in the model by treating them as either symmetric or asymmetric ellipsoids. For non-spherical molecules, $f>1$ and the magnitude of the deviation from unity describes the degree of non-spherical nature of the solute molecule. In (1), the parameter $C$ signifies the extent of coupling between the solute and the solvent and is known as the boundary condition parameter or the solute-solvent coupling parameter and 
the two limiting cases are the hydrodynamic stick and slip. ${ }^{56}$ For a non-spherical solute molecule, the value of $C$ follows the inequality, $0<C \leq 1$ and the exact value of $C$ is determined by the axial ratio of the ellipsoid under consideration. ${ }^{56}$ The SED theory works reasonably well when the intermolecular forces between the solute and solvent are repulsive. However, the SED theory breaks down when the solute and solvent experience attractive intermolecular interactions. The breakdown of SED theory is manifested in the measured reorientation times of the solutes, which are longer than the predicted ones. The solutes DMDPP and DPP have been treated as asymmetric ellipsoids and the reorientation times with slip and stick boundary condition were calculated. The details of these calculations have been presented elsewhere. ${ }^{57}$

Since the reorientation times of the solutes are proportional to the solvent viscosity at a given temperature, they are plotted as a function of $\eta / T$ in figure 3. The SED slip and stick lines which were calculated by treating the solutes as asymmetric ellipsoids are also presented in the figure. It is evident from the figure that the reorientation times of DMDPP and DPP lie on either side of the slip line. In other words, DMDPP and DPP experience subslip and super-slip behaviour in $\left[\mathrm{bmim}^{+}\right]\left[\mathrm{PF}_{6}^{-}\right]$, respectively. Numerous studies carried out with dipolar

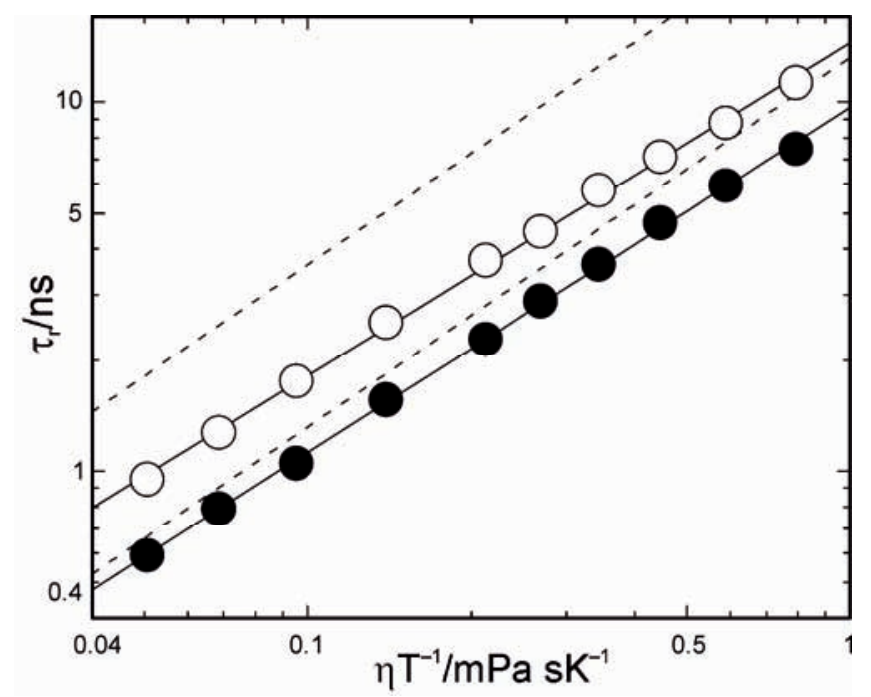

Figure 3. Plots of $\tau_{r}$ vs. $\eta / T$ for DMDPP (•) and DPP (O) in $\left[\mathrm{bmim}^{+}\right]\left[\mathrm{PF}_{6}^{-}\right]$. The solid lines through the experimental data points were obtained by linear least squares fit. The upper and lower dashed lines are the SED stick and slip lines, respectively. Reprinted with permission from ref. 46. Copyright (2005) American Institute of Physics. and non-polar solutes in non-polar solvents indicate that the rotation of a medium-sized solute molecule is close to slip behaviour. ${ }^{50,58-69}$ Thus, the rotation of both DMDPP and DPP is in accordance with the predictions based on the literature data. However, structurally these two solutes are similar and their van der Waals volumes are almost the same. ${ }^{57}$ Hence, according to SED theory they are expected to have identical reorientation times in a given solvent. In fact, a completely opposite trend has been observed; rotation of DPP is slower by a factor of 1.5-1.6 compared to DMDPP. This observation indicates that the solute DPP is indeed experiencing strong specific interactions with the anion of the ionic liquid $\left[\mathrm{bmim}^{+}\right]\left[\mathrm{PF}_{6}^{-}\right]$and as a consequence, its rotation is hindered compared to DMDPP. The discussion presented here demonstrates that ionic liquids do experience specific interactions with the organic solutes despite their strong cohesive nature. Thus the logical question that can be asked is: Why other organic solutes used in the earlier studies did not experience specific interactions with ionic liquids such that their rotation is hindered? The probable reason is the hydrogen bonding nature of DPP, which is very different, compared to other organic solutes. From the $a b$ initio molecular orbital calculations it has been established that DPP forms different kinds of aggregates, such as stack dimer, H-bond dimer, translation dimer, stack-trimer and H-bond trimer in the solid state. It has been shown that $\mathrm{H}$-bond dimer is about $100 \mathrm{~kJ} \mathrm{~mol}^{-1}$ more stable than the monomer. ${ }^{70}$ Because of the stability of these aggregates; DPP needs to form reasonably strong hydrogen bonds with the solvent so that the monomer form is soluble. Moreover, the solubility of DPP also depends on the hydrogen bond accepting ability of the solvent. For example, DPP interacts more strongly with $n$ alcohols compared to glycerol because the intermolecular hydrogen bonding is weaker in the former compared to the latter. As a consequence, the ratio of the reorientation times of DPP to DMDPP, which also represents the strength of the interaction between DPP and its surroundings, is $2-3$ in alcohols whereas it is only about 1.5 in glycerol. In contrast, a majority of organic solutes including the charged ones are soluble in conventional solvents as well as in ionic liquids even if they do not experience specific interactions with their surroundings. This is the most probable reason as to why the solutes examined so far did not display unusual rotational behaviour in ionic liquids compared to conventional solvents. In essence, this preliminary investigation 
illustrates that organic solutes do experience specific interactions with the constituents of the ionic liquid despite the strong cohesive nature of these liquids.

\subsection{Interplay of size effects and specific interactions}

As already mentioned in the introduction, rotational diffusion of charged solutes in ionic liquids has not been investigated extensively so far. To understand the influence of ionic interactions between the solute and the solvent on solute rotation, rotational diffusion of an ionic solute needs to be examined in an ionic liquid. The role of ionic interactions can be better appreciated by comparing the rotational diffusion of an ionic solute with that of a structurally similar non-polar analogue of comparable volume. In other words, rotational diffusion of two structurally similar solutes of comparable size but possessing different functional groups needs to be studied. Besides this aspect, one can also investigate the influence of solvent size on solute rotation, which leads to the incidence of size effects, by choosing a solvent that has different van der Waals volume, compared to the ionic liquid. Such a judicious choice of the solute and solvent pair will enable us to discern the above-mentioned effects (size effects and specific interactions) because the hydrodynamic friction experienced by the solutes will be similar. For this purpose, the solutes 9-phenylanthracene (9PA) and rhodamine 110 (R110) were chosen since they fulfill the requisite criteria and their molecular structures are displayed in figure 4.

It is evident from the figure that the two solutes are similar except for the functional groups present on R110. The solute R110 possesses two terminal $\mathrm{NH}_{2}$ groups, a carboxylic group and is positively charged, thus it is likely to form strong hydrogen bonds and also experience ionic interactions with

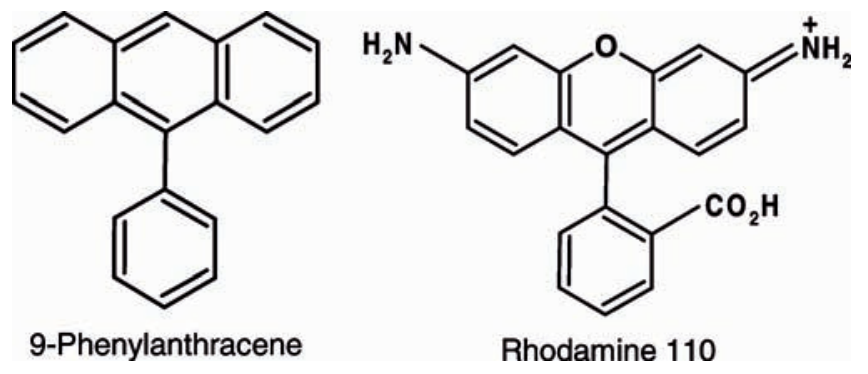

Figure 4. Molecular structures of the solutes 9-phenylanthracene and rhodamine 110 .
$\left[\mathrm{bmim}^{+}\right]\left[\mathrm{PF}_{6}^{-}\right]$. The solvents chosen for the present study are $\left[\mathrm{bmim}^{+}\right]\left[\mathrm{PF}_{6}^{-}\right]$and glycerol, which are highly associative and viscous. In addition to having dissimilar functional groups, the sizes of these solvents are also different. The van der Waals volume of glycerol is nearly three times smaller than that of $\left[\mathrm{bmim}^{+}\right]\left[\mathrm{PF}_{6}^{-}\right]{ }^{46}$ Thus, these two solvents are well suited for exploring the interplay of size effects and ionic interactions. This study, essentially deals with the measurement of reorientation times of the solutes 9PA and R110 in $\left[\mathrm{bmim}^{+}\right]\left[\mathrm{PF}_{6}^{-}\right]$and glycerol as a function of temperature. The measured reorientation times of both the solutes in the two solvents have been compared under isoviscous conditions and also with the calculated ones obtained using hydrodynamic and quasihydrodynamic theories. To compare the experimentally measured reorientation times with the hydrodynamic predictions, $\tau_{r}$ vs $\eta / T$ plots for 9PA and R110 in the two solvents are presented in figure 5. Even though 9PA and R110 are structurally similar, their axial radii and van der Waals volumes are not identical. Thus, the reorientation times of R110 calculated using the SED theory should be longer compared to those of 9PA by a factor of 1.7 and 1.4 with the slip and stick boundary conditions, respectively. The details of these calculations have been presented in our recent publication. ${ }^{47}$ It is evident from the figure that the reorientation times of 9PA in ionic liquid and glycerol are closer to the predictions of slip hydrodynamics whereas those of R110 follow stick behaviour. However, the important feature to be noted from the figure is that the reorientation times of R110 are longer by a factor of 7-8 compared to $9 \mathrm{PA}$ in $\left[\mathrm{bmim}^{+}\right]\left[\mathrm{PF}_{6}^{-}\right]$whereas this number is only in the range of 3-4 in glycerol. These differences are much larger than the ones predicted by the SED theory. What are the reasons for this observed behaviour? The longer reorientation times of R110 compared to 9PA are as a consequence of specific interactions between R110 and the solvents. The larger differences observed between reorientation times of 9PA and R110 in $\left[\mathrm{bmim}^{+}\right]\left[\mathrm{PF}_{6}^{-}\right]$ compared to glycerol are perhaps due to ionic interactions between the solute and the anion $\left[\mathrm{PF}_{6}^{-}\right]$. To get a better appreciation of the differences in the rotational diffusion of a particular solute in the two solvents, the reorientation times of 9PA and R110 in glycerol were normalized with respect to the viscosity of the ionic liquid at each temperature by multiplying with a normalization factor, $\gamma=\left(\eta_{\text {ionic }}\right.$ liquid $/$ $\left.\eta_{\text {glycerol }}\right)_{\gamma}$. The reorientation times of 9PA and R110 in glycerol normalized in this manner along with 

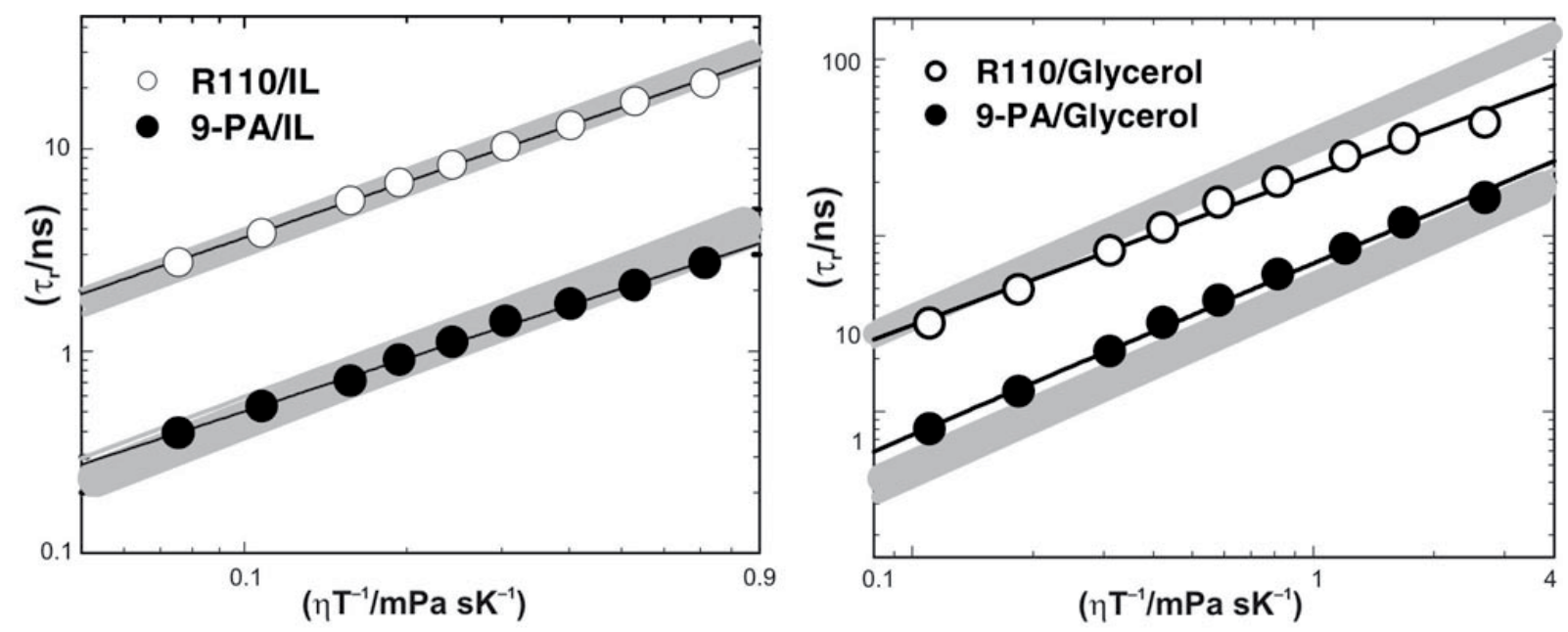

Figure 5. Plots of $\tau_{r}$ vs $\eta / T$ for 9PA $(\bullet)$ and $\mathrm{R} 110(\mathrm{O})$ in $\left[\mathrm{bmim}^{+}\right]\left[\mathrm{PF}_{6}^{-}\right]$and glycerol. The upper and lower gray coloured bands represent theoretically calculated slip and stick boundary conditions. The lower and upper limits of each band represent the reorientation times of 9PA and R110, respectively, for a particular boundary condition (see text for details).

those in $\left[\mathrm{bmim}^{+}\right]\left[\mathrm{PF}_{6}^{-}\right]$are plotted as a function of $\eta / T$ in figures 6 and 7, respectively. Inspection of figure 6 reveals that the normalized reorientation times of 9PA are longer by a factor of 1.4-1.6 in glycerol compared to $\left[\mathrm{bmim}^{+}\right]\left[\mathrm{PF}_{6}^{-}\right]$. On the other hand, an opposite trend can be noticed in the rotational diffusion of R110 upon the inspection of figure 7.

The SED theory predicts that the rotational diffusion of a solute molecule in the absence of specific and non-specific interactions is solely governed by solvent viscosity. Based on this perception, it is not evident as to why the viscosity normalized reorientation times of 9PA are longer in glycerol compared to $\left[\mathrm{bmim}^{+}\right]\left[\mathrm{PF}_{6}^{-}\right]$. Since 9PA is a non-polar solute, the only reason for the observed behaviour is the disparity in the sizes of the two solvents, which leads to the prevalence of size effects. It has been well established that in the absence of specific interactions, an increase in the solvent size results in a decrease in the friction experienced by the solute molecule. In the present case, the van der Waals volume of glycerol is about three times smaller than that of ionic liquid ${ }^{46}$ and hence it offers more friction to the solute 9PA. Quasihydrodynamic theories of Gierer-Wirtz $(\mathrm{GW})^{71}$ and Dote-Kivelson-Schwartz $(\mathrm{DKS})^{72}$ have often been used to rationalize these kinds of experimental results. ${ }^{59-65}$ According to the GW and DKS theories, the extent of solute-solvent coupling depends on the relative sizes of the solute and solvent, which is not taken into consideration by the SED hydrodynamic theory. The GW theory visualizes the solute to be surrounded by concentric shells of solvent and the boundary condition parameter, $C$ is obtained by considering how the angular velocity of the solvent molecules in the successive shells surrounding the solute decreases as a function of the distance away from it. On the other hand, the DKS theory takes into account the free volume of the solvent as well while calculating the boundary condition parameter.

Thus, GW and DKS theories have been applied to rationalize the rotational diffusion of 9PA in $\left[\mathrm{bmim}^{+}\right]$ $\left[\mathrm{PF}_{6}^{-}\right]$and glycerol and the reorientation times obtained using these theories are also plotted in figure 6 . Even though both the theories are successful in reproducing the experimental trend in a qualitative manner, there is a better agreement between the GW theory and the experimental results. It must be noted that the highly associative nature of the two solvents is not responsible for the lack of quantitative agreement between the experimental results and the numbers obtained with GW and DKS models. Even in case of alcohols and alkanes a similar pattern has been noticed for a number of non-polar solutes. ${ }^{48,58-62}$ The results of 9PA in the two solvents indicate that the mechanical friction experienced by the solute molecule is also dependent on the size of the solvent.

In an attempt to incorporate this feature and estimate the mechanical friction experienced by R 110 in $\left[\mathrm{bmim}^{+}\right]\left[\mathrm{PF}_{6}^{-}\right]$and glycerol, experimentally meas- 
ured reorientation times of 9PA in these two solvents were multiplied by a factor 1.36 , which was obtained by taking the ratio of $V_{\mathrm{R} 110} f_{\mathrm{R} 110}$ and $V_{9 \mathrm{PA}}$ $f_{9 \mathrm{PA}}$. In this estimation, the boundary condition parameter, $C$ was not used because it is a solvent dependent parameter. The mechanical friction calculated in this manner for R110 in $\left[\mathrm{bmim}^{+}\right]\left[\mathrm{PF}_{6}^{-}\right]$and glycerol is represented by dotted and dashed lines, respectively, in figure 7. But the experimentally measured reorientation times of R110 in $\left[\mathrm{bmim}^{+}\right]$ $\left[\mathrm{PF}_{6}^{-}\right]$and glycerol (normalized reorientation times) are longer than the numbers obtained in this manner by a factor of 5.5 and 2.5 , respectively. This exercise firmly establishes that mechanical friction alone cannot account for the rotational diffusion of R 110 in $\left[\mathrm{bmim}^{+}\right]\left[\mathrm{PF}_{6}^{-}\right]$and glycerol. The significantly longer reorientation times of R110 in the two solvents are an indication that the solute molecule is indeed experiencing strong specific interactions with $\left[\mathrm{bmim}^{+}\right]\left[\mathrm{PF}_{6}^{-}\right]$and glycerol. Since R110 has two terminal amino groups and a carboxylic group, it can form hydrogen bonds with the hydroxyl groups of glycerol. Moreover, because of its charged character it can also experience specific interactions with the anion $\left[\mathrm{PF}_{6}^{-}\right]$of the ionic liquid through hydrogen

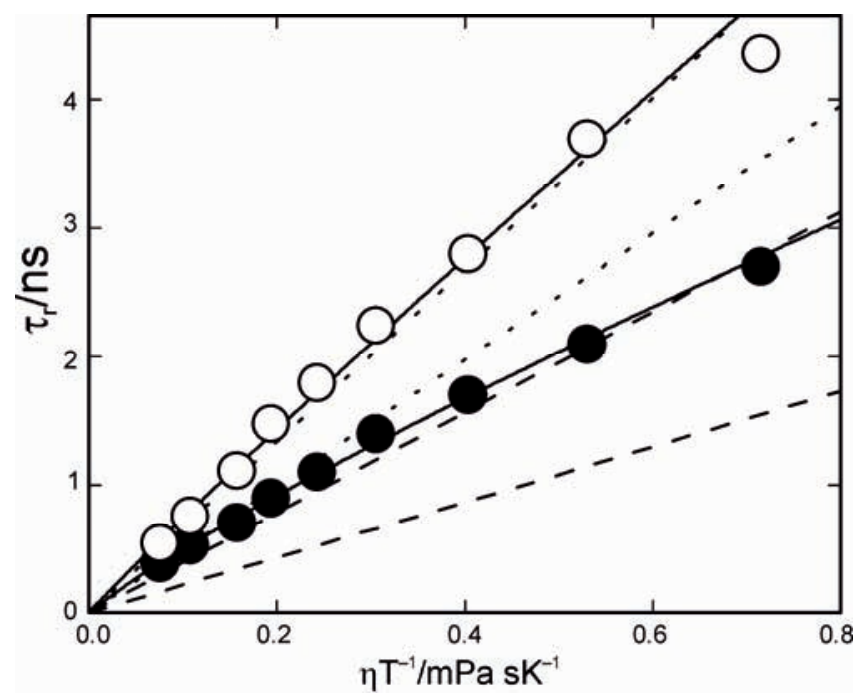

Figure 6. Comparison of the reorientation times of 9PA in $\left[\mathrm{bmim}^{+}\right]\left[\mathrm{PF}_{6}^{-}\right](\bullet)$ and glycerol $(\bigcirc)$. The lines passing through the data points are the fitted ones obtained from $\log -\log$ plots of $\tau_{r}$ vs. $\eta / T$. Reorientation times calculated with $\mathrm{GW}$ model for $9 \mathrm{PA} /\left[\mathrm{bmim}^{+}\right]\left[\mathrm{PF}_{6}^{-}\right]$and $9 \mathrm{PA} /$ glycerol are represented by the dotted lines whereas the corresponding reorientation times obtained with the DKS model are given by dashed lines. Reprinted with permission from ref. 47. Copyright (2008) American Institute of Physics. bonding and ion pair formation. It has been established that specific interactions influence rotational diffusion only if hydrogen-bonding dynamics takes place on a time scale that is comparable to or slower than the time scale of rotational diffusion, which in turn is determined by the strength of the solutesolvent hydrogen bond ${ }^{50}$ If there is some flexibility of the hydrogen bonds to angular motion, the smallstep angular displacement occurring during the lifetime of a hydrogen bond will not cause any solvent to be dragged along, thus there will be no effect on rotational diffusion of the solute. ${ }^{73,74}$ On the other hand, if the solute-solvent hydrogen bonds are strong enough, they will remain intact during the course of the rotation. In other words, stronger solute-solvent hydrogen bonds lead to slower rotation of the solute. In fact, this hypothesis has been verified in case of the rotational diffusion of DMDPP and DPP in ethanol and trifluoroethanol and also in isomeric butanols by correlating the measured reorientation times with the calculated solute-solvent interaction strengths. ${ }^{54,75}$ In the present study, despite experiencing higher mechanical friction in glycerol, which is evident from the calculation presented earlier, the viscosity normalized reorientation times of R110 in

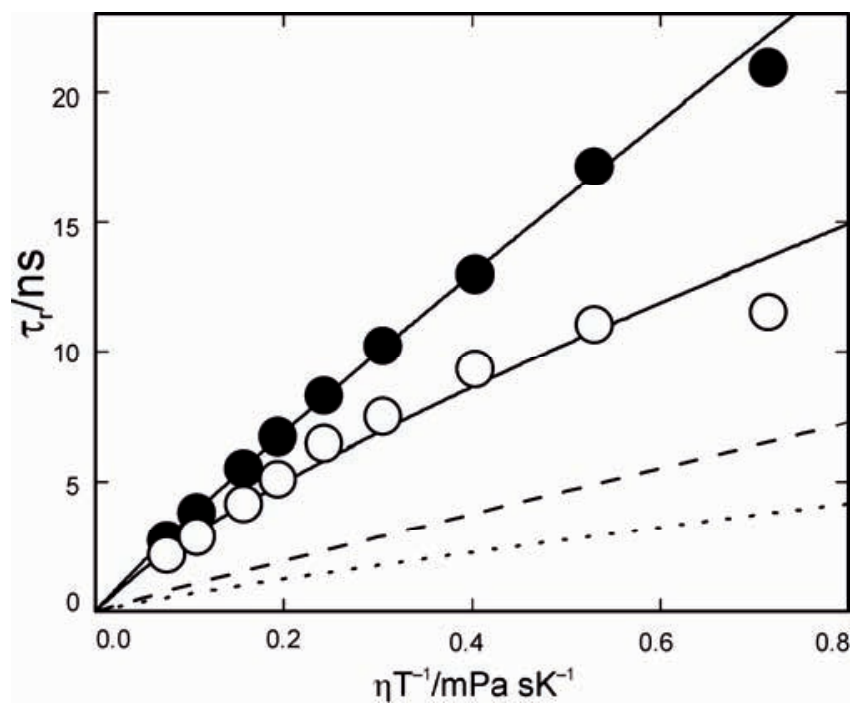

Figure 7. Comparison of the reorientation times of $\mathrm{R} 110$ in $\left[\mathrm{bmim}^{+}\right]\left[\mathrm{PF}_{6}^{-}\right](\bullet)$ and glycerol $(O)$. The lines passing through the data points are the fitted ones obtained from $\log -\log$ plots of $\tau_{r}$ vs $\eta / T$. The dotted and dashed lines represent the mechanical friction experienced by $\mathrm{R} 110$ in $\left[\mathrm{bmim}^{+}\right]\left[\mathrm{PF}_{6}^{-}\right]$and glycerol, respectively. These lines were obtained from the measured reorientation times of 9PA in the two solvents (see text for details). Reprinted with permission from ref. 47 . Copyright (2008) American Institute of Physics. 
glycerol are shorter than the ones measured in $\left[\mathrm{bmim}^{+}\right]\left[\mathrm{PF}_{6}^{-}\right]$. This result clearly indicates that the interactions between $\mathrm{R} 110$ and $\left[\mathrm{bmim}^{+}\right]\left[\mathrm{PF}_{6}^{-}\right]$are stronger compared to ones between R110 and glycerol.

\section{Photoisomerization studies}

The rotational diffusion studies presented in the previous section indicate that organic solutes do experience specific interactions with ionic liquids such that their rotation is hindered. As mentioned in the introduction, photoisomerization is another process, which is affected by the friction at the molecular level. Having comprehended rotational diffusion process in $\left[\mathrm{bmim}^{+}\right]\left[\mathrm{PF}_{6}^{-}\right]$, we now turn our attention to the process of photoisomerization. As mentioned in the introduction section, investigations dealing with photoisomerization of olefins and polyenes in ionic liquids have begun only recently. ${ }^{29,43-45}$ Nevertheless, the effect of high viscosities of these ionic liquids, which arises due to the strong interactions between the cations and anions that constitute them, on photoisomerization, has not been addressed explicitly. Moreover, besides the viscosity and polarity of the medium, specific interactions between the solute undergoing isomerization and the solvent are also known to facilitate isomerization by lowering the barrier height. In order to understand the role of viscosity and specific interactions on the photoisomerization process, we have initiated isomerization studies in $\left[\mathrm{bmim}^{+}\right]\left[\mathrm{PF}_{6}^{-}\right]$. The main objective of this study is to establish whether or not specific interactions between the solute and the ionic liquid alter the rate of photoisomerization process. In the work presented below, photoisomerization rate constants of two cyanine derivatives have been measured in $\left[\mathrm{bmim}^{+}\right]\left[\mathrm{PF}_{6}^{-}\right]$and are compared with those obtained in a conventional organic solvent, which is isoviscous.

A brief introduction to the process of photoisomerization of olefins and polyenes is imperative prior to the discussion on the experimental findings. Photoisomerization of olefins and polyenes such as stilbene and its analogues, ${ }^{76}$ diphenylbutadienes ${ }^{77-82}$ and carbocyanines ${ }^{83-92}$ has been thoroughly investigated in organic solvents in the recent past. Figure 8 shows a schematic of the isomerization process for olefins and polyenes. The excited state isomerization of these systems essentially involves an activated twist motion about the double bond/bonds to an intermediate geometry. In the twisted geometry, the excited state becomes close in energy to the ground state thus it rapidly decays to the twisted ground electronic state by internal conversion. Once on the ground state surface, branching between the isomer and the normal form occurs. The nonradiative process is dominated by the barrier crossing or the twisting motion because the internal conversion from the twisted excited state is very rapid. In essence, the non-radiative rate has been identified as the rate of photoisomerization in these systems. Since a large amplitude motion is involved in the barrier crossing, the non-radiative rate depends on solute-solvent frictional coupling. In other words, isomerization rate constants are sensitive to the viscosity of the medium. However, when the solute is solubilized in a very high viscous medium such as a polymer matrix, the twisting motion gets inhibited and internal conversion becomes the main nonradiative process. ${ }^{85}$ Besides the viscosity of the medium, solute-solvent dielectric interactions also govern the photoisomerization rate due to the polar nature of the transition state. Kramers' theory ${ }^{93}$ has been widely applied to understand the isomerization rates in terms of barrier heights. ${ }^{50,51,76}$ Although Kramers' theory has been quite successful, systematic deviations from Kramers' model were observed,

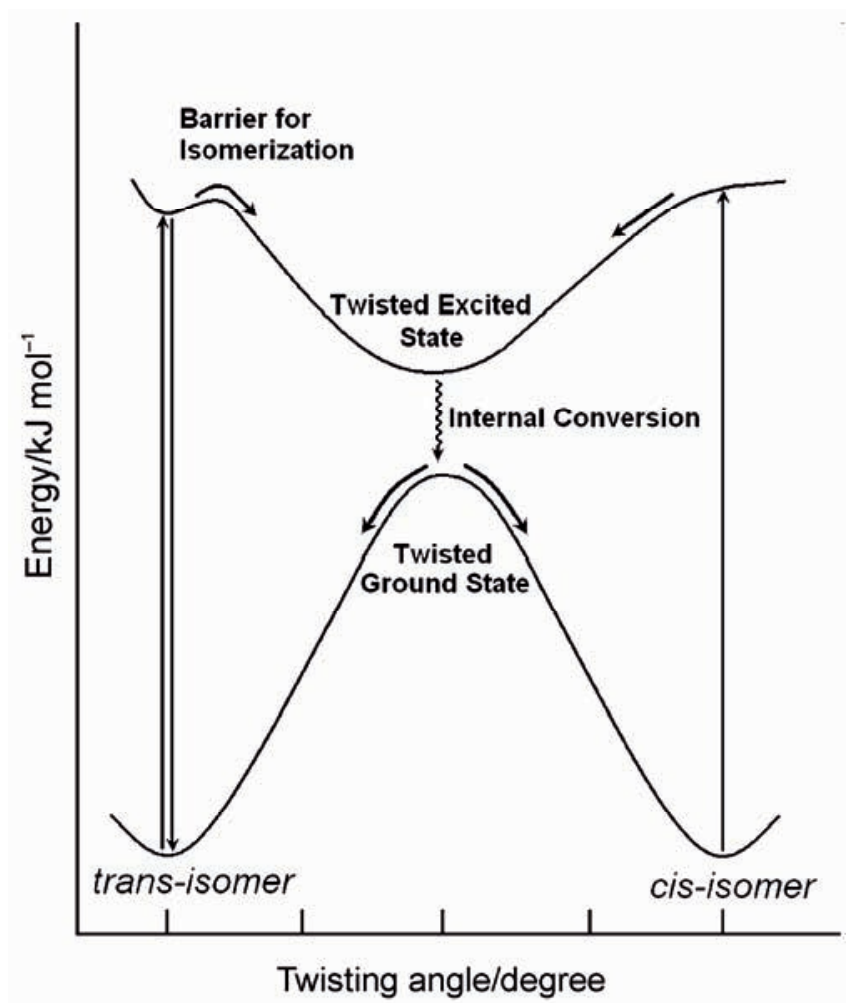

Figure 8. Schematic for the excited state isomerization of olefins and polyenes. 
which have been rationalized on the basis of microscopic friction being different from the bulk viscosity of the solvent, frequency-dependent frictional effects in which solute-solvent couplings depend on the relative time scales of the solute and solvent isomerization motions and inadequacy of the onedimensional model.

From the preliminary photoisomerization studies carried out in ionic liquids, the role of specific solute-solvent interactions on this process is not apparent. In order to shed light on this aspect, we have examined the process of photoisomerization of two cyanine derivatives, 3,3'-diethyloxadicarbocyanine iodide (DODCI) and merocyanine 540 (MC 540) in $\left[\mathrm{bmim}^{+}\right]\left[\mathrm{PF}_{6}^{-}\right]$over the temperature range $293-343 \mathrm{~K}$. Figure 9 gives molecular structures of the two probes and it is evident from the figure that DODCI is a cationic solute whereas MC 540 is anionic. The results obtained in $\left[\mathrm{bmim}^{+}\right]\left[\mathrm{PF}_{6}^{-}\right]$have been compared with those in aqueous glycerol $(93 \mathrm{wt} \%$ glycerol $+7 \mathrm{wt} \%$ water). Aqueous glycerol has been chosen because its viscosity is identical to $\left[\mathrm{bmim}^{+}\right]$ $\left[\mathrm{PF}_{6}^{-}\right]$but has higher polarity compared to the ionic liquid. Moreover, because of the distinct chemical nature of these solvent molecules, their ability to experience specific interactions with the probes will not be the same, which can also influence the photoisomerization rates significantly. Since the nonradiative rate constant $\left(k_{n r}\right)$ has been identified as the rate of isomerization for these systems, the measured quantum yields $\left(\phi_{f}\right)$ and fluorescence lifetimes

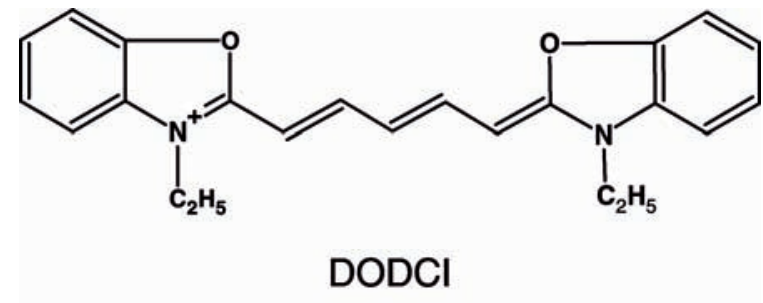

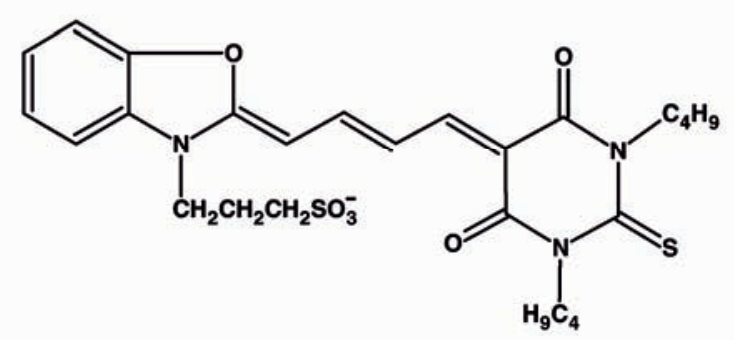

MC 540

Figure 9. Molecular structures of the solutes DODCI and MC 540. $\left(\tau_{f}\right)$ of DODCI and MC 540 in $\left[\mathrm{bmim}^{+}\right]\left[\mathrm{PF}_{6}^{-}\right]$and aqueous glycerol were employed to obtain this parameter. Fluorescence quantum yield and lifetime are related to radiative $\left(k_{r}\right)$ and non-radiative rate constants by the following relations: ${ }^{94}$

$$
\begin{gathered}
\phi_{f}=\frac{k_{r}}{k_{r}+k_{n r}}, \\
\tau_{f}=\frac{1}{k_{r}+k_{n r}} .
\end{gathered}
$$

Radiative rate constants were obtained from the measured quantum yields and lifetimes using (2) and (3). Non-radiative rate constants of DODCI and MC 540 in $\left[\mathrm{bmim}^{+}\right]\left[\mathrm{PF}_{6}^{-}\right]$and aqueous glycerol were obtained using the parameters $k_{r}$ and $\tau_{f}$. Since the variation of photoisomerization rate constant with
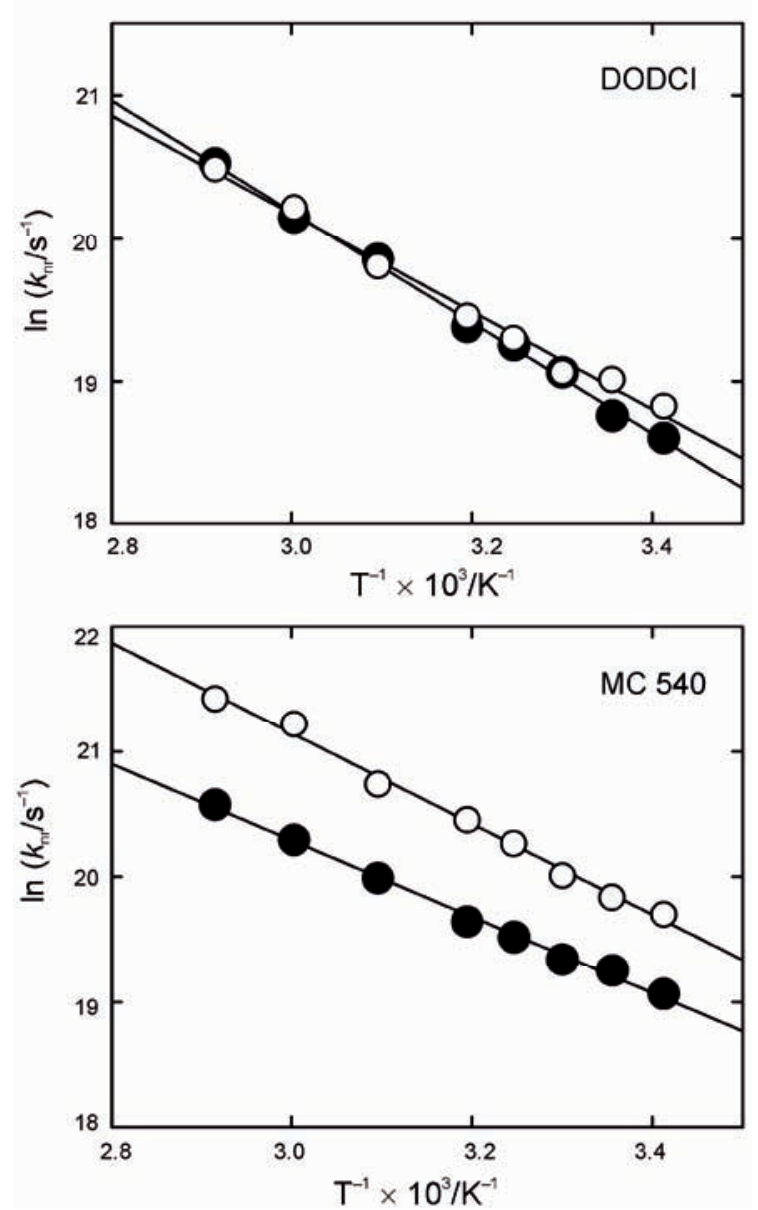

Figure 10. Plots of $\ln \left(k_{n r}\right)$ vs $1 / T$ for DODCI and MC 540 in $\left[\mathrm{bmim}^{+}\right]\left[\mathrm{PF}_{6}\right](\bullet)$ and aqueous glycerol $(O)$. The lines passing through the data points were obtained by linear least-squares fit. Reprinted with permission from ref. 48. Copyright (2008) American Institute of Physics. 
temperature follows Arrhenius-type relation, the parameters $\ln \left(k_{n r}\right)$ and $1 / T$ were plotted as ordinate and abscissa, respectively, in figure 10 for two probes in $\left[\mathrm{bmim}^{+}\right]\left[\mathrm{PF}_{6}^{-}\right]$and aqueous glycerol. It is evident from the figure that $k_{n r}$ values of DODCI are almost identical in aqueous glycerol and $\left[\mathrm{bmim}^{+}\right]\left[\mathrm{PF}_{6}^{-}\right]$over the temperature range $293-343 \mathrm{~K}$. This result indicates that the viscosity is the sole parameter responsible for controlling the rate of photoisomerization of DODCI in these two solvents. It must also be noted that despite having vastly different polarities and chemical nature, both aqueous glycerol and $\left[\mathrm{bmim}^{+}\right]\left[\mathrm{PF}_{6}^{-}\right]$offer identical friction to the photoisomerization process of DODCI.

At this juncture it is imperative to compare the results obtained by Chakrabarty et $a l^{29}$ for the isomerization of DODCI in $\left[\mathrm{bmim}^{+}\right]\left[\mathrm{PF}_{6}^{-}\right]$. In their study, the lifetimes and quantum yields of DODCI in $\left[\mathrm{bmim}^{+}\right]\left[\mathrm{PF}_{6}^{-}\right]$were measured with varying amounts of water and it has been observed that the rate of photoisomerization increases with an increase in the water content, which has been ascribed to the decrease in the viscosity of the medium on the addition of water. However, addition of water to $\left[\mathrm{bmim}^{+}\right]\left[\mathrm{PF}_{6}^{-}\right.$ ] also increases the polarity of the medium besides decreasing the viscosity. The quantum yield values reported for DODCI in $\left[\mathrm{bmim}^{+}\right]\left[\mathrm{PF}_{6}^{-}\right]$at $298 \mathrm{~K}$ in the two studies are comparable, $\phi_{f}=0.93$ (ref. 28) and $\phi_{f}=0.76$ (ref. 48). In contrast, the lifetimes reported for DODCI in these two studies are vastly different. Chakrabarty et $a l^{29}$ report a value of $3.64 \mathrm{~ns}$ for DODCI in $\left[\mathrm{bmim}^{+}\right]\left[\mathrm{PF}_{6}^{-}\right]$at $298 \mathrm{~K}$, whereas our measurements gave a value of $1.67 \mathrm{~ns}$. What is the reason for this anomaly? The most likely reason for this discrepancy is the excitation wavelengths employed in the two studies. DODCI was excited at $575 \mathrm{~nm}$ in this study whereas the wavelength of excitation used by Chakrabarty et $a l^{29}$ is $408 \mathrm{~nm}$. It has been reported in literature ${ }^{95}$ and we have also noticed during our experiments, that $\left[\mathrm{bmim}^{+}\right]\left[\mathrm{PF}_{6}^{-}\right]$displays weak emission in the wavelength region 300 $600 \mathrm{~nm}$ and the emission maxima shifts to longer wavelengths with an increase in the excitation wavelength. This kind of emission is observed even from rigorously purified samples, which has been ascribed to various associated forms of imidazolium ions. ${ }^{95}$ To find out if the different excitation wavelengths employed in the two studies are indeed the reason for the discrepancies observed, the emission spectra of neat $\left[\mathrm{bmim}^{+}\right]\left[\mathrm{PF}_{6}^{-}\right]$were recorded in the wavelength range $570-700 \mathrm{~nm}$ by exciting at 408 as well as $555 \mathrm{~nm}$ and the results are presented in fig- ure 11. It is evident from the figure that the integrated area of the spectrum when excited at $408 \mathrm{~nm}$ is 3.7 times larger compared to the one obtained at $555 \mathrm{~nm}$ excitation. Moreover, at $600 \mathrm{~nm}$, where the emission was monitored for the lifetime measurements, the emission intensity of $\left[\mathrm{bmim}^{+}\right]\left[\mathrm{PF}_{6}^{-}\right]$is a factor of 4.2 higher when the ionic liquid was excited at $408 \mathrm{~nm}$ compared to $555 \mathrm{~nm}$ excitation. It must also be noted that $555 \mathrm{~nm}$ excitation was employed only for the quantum yield measurements of DODCI and for the lifetime measurements $575 \mathrm{~nm}$ excitation was used. The ratio of emission intensity of $\left[\mathrm{bmim}^{+}\right]\left[\mathrm{PF}_{6}^{-}\right]$at $600 \mathrm{~nm}$ for $408 \mathrm{~nm}$ to $575 \mathrm{~nm}$ excitation is more than a factor of 8 . In other words, when $408 \mathrm{~nm}$ was employed to excite DODCI in $\left[\mathrm{bmim}^{+}\right]\left[\mathrm{PF}_{6}^{-}\right]$, emission from the ionic liquid strongly interferes with the emission of the probe. Besides, the absorbance of DODCI in $\left[\mathrm{bmim}^{+}\right]\left[\mathrm{PF}_{6}^{-}\right]$at $408 \mathrm{~nm}$ is lower by more than a factor of 10 compared to the one at $575 \mathrm{~nm}$. Hence, a combination of these two factors increases the emission contribution from the ionic liquid by a factor of 80 upon excitation with $408 \mathrm{~nm}$ compared to $575 \mathrm{~nm}$. To substantiate these arguments, the lifetime of DODCI in $\left[\mathrm{bmim}^{+}\right]\left[\mathrm{PF}_{6}^{-}\right]$ was measured by exciting the sample at $408 \mathrm{~nm}$, which gave a value of $4.0 \mathrm{~ns}$ and this number is close to the one reported by Chakrabarty et al. ${ }^{29}$ Thus, the observed discrepancies between the present work and those reported in ref. 29 are a consequence of different excitation wavelengths employed

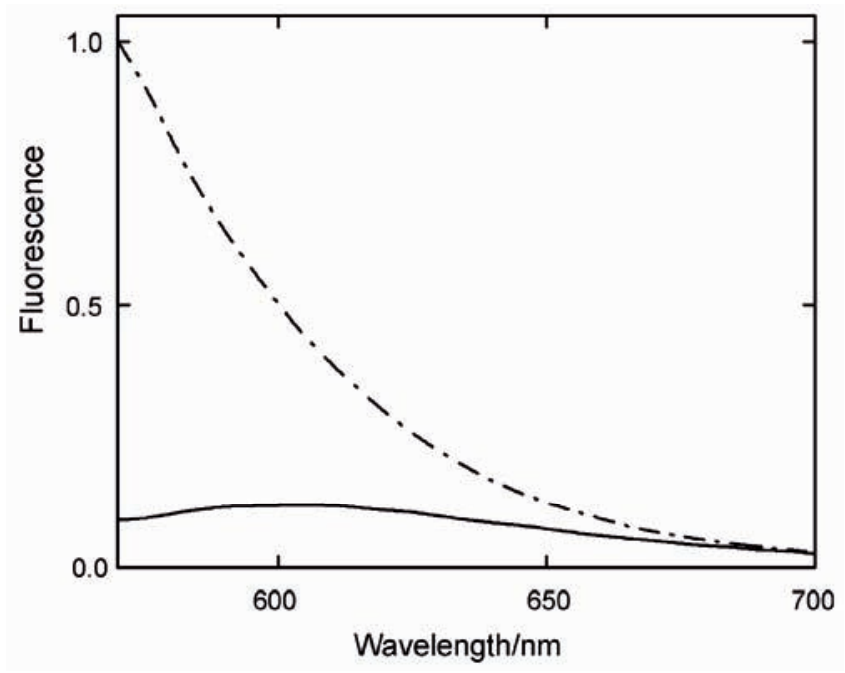

Figure 11. Normalized emission spectra of neat $\left[\mathrm{bmim}^{+}\right]\left[\mathrm{PF}_{6}^{-}\right]$at room temperature upon excitation at $408 \mathrm{~nm}$ (broken line) and $555 \mathrm{~nm}$ (solid line). Reprinted with permission from ref. 48. Copyright (2008) American Institute of Physics. 
in the two studies. In essence, the vast amount of literature available on the photophysical studies involving DODCI and also the arguments presented here indicate that $550-600 \mathrm{~nm}$ region is appropriate for its excitation, especially in $\left[\mathrm{bmim}^{+}\right]\left[\mathrm{PF}_{6}^{-}\right]$.

In contrast to DODCI, $k_{n r}$ values of MC 540 in aqueous glycerol are about a factor of 2 higher compared to the ones in ionic liquid throughout the temperature range studied. This result is surprising considering the fact that both DODCI and MC 540 are ionic polyenes but only the latter shows marked differences in the isomerization rate constants in the two solvents. A similar result has been obtained by Onganer $e t a l^{91}$ who observed that the isomerization rate constant of MC 540 is a factor of 5 higher in 1propanol compared to nonanenitrile at $298 \mathrm{~K}$ despite identical viscosities of both the solvents. This result indicates that solvent polarity plays an important role in the photoisomerization of MC 540. Since the polarity of aqueous glycerol is considerably higher than $\left[\mathrm{bmim}^{+}\right]\left[\mathrm{PF}_{6}^{-}\right]$, photoisomerization of $\mathrm{MC} 540$ proceeds faster in aqueous glycerol. To understand the effect of solute-solvent frictional coupling on photoisomerization rates, the following relation has often been used.

$$
K_{\text {iso }}=F(\zeta) \exp \left(-E_{0} / R T\right) \text {. }
$$

In the above equation, $E_{0}$ is the intrinsic barrier height and $R$ is the universal gas constant. The term, $F(\zeta)$ is a dynamical quantity that depends on solute-solvent frictional coupling. ${ }^{91}$ The solute-solvent frictional effects and the viscosity dependence of the isomerization rates are usually compared to theoretical models for $F(\zeta)$. Even though $\ln \left(k_{n r}\right)$ vs $1 / T$ plots are given in figure 10 , the activation energies or barrier heights of the reaction cannot be estimated from such plots because of the expected influence of temperature on viscosity. For this purpose, isoviscous plots in a homologous series of solvents are often employed. Since the transition states involved in isomerization of DODCI and MC 540 have a polar character, solvent polarity also influences isomerization rate constants. To disentangle the effects of viscosity and polarity on isomerization rate constants and obtain the activation energies of the reaction, non-radiative rate constants were measured under isoviscous and isodielectric conditions and modified Kramers' equation has been employed. ${ }^{51}$ However, in the present study, only two solvents of different nature were used and hence the application of theoretical models to understand solute-solvent fric- tional coupling is not possible. Despite this limitation, the significant differences observed in the photoisomerization rate constants of MC 540 in $\left[\mathrm{bmim}^{+}\right]\left[\mathrm{PF}_{6}^{-}\right]$and aqueous glycerol can be understood in a qualitative manner with the help of static solvation effects such as the solute-solvent hydrogen bonding.

Apart from the structure given in figure 9, MC 540 exists in zwitterionic resonance structures ${ }^{91}$ as shown in figure 12. Hydrogen bonding between the hydroxyl groups of aqueous glycerol and the carbonyl groups stabilizes the negative charge on the carbonyl oxygens, which lowers the energy of the zwitterionic structures and hence the barrier for isomerization is decreased. This explanation is consistent with the one given by Onganer et $a l^{91}$ while rationalizing the higher isomerization rate constants obtained for MC 540 in alcohols compared to nitriles. Although the results obtained for DODCI and MC 540 in a hydroxyl group containing solvent such as aqueous glycerol appear to be along the expected lines, it was not evident whether or not these two probes experience specific interactions with ions that constitute the ionic liquid, $\left[\mathrm{bmim}^{+}\right]\left[\mathrm{PF}_{6}^{-}\right]$. From this study, it has been established that both DODCI and MC 540 do not experience specific interactions with $\left[\mathrm{bmim}^{+}\right]\left[\mathrm{PF}_{6}^{-}\right]$and the photoisomerization of these probes is solely governed by the viscosity of the medium.

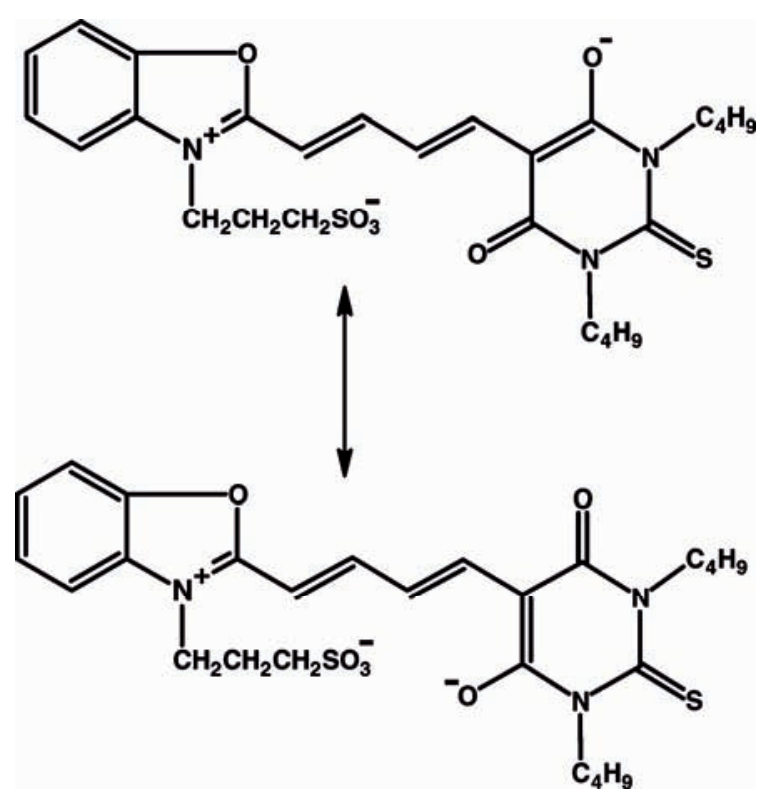

Figure 12. Zwitterionic resonance structures of $\mathrm{MC}$ 540 . 
To find an experimental evidence for this hypothesis, rotational diffusion measurements of the two solutes in $\left[\mathrm{bmim}^{+}\right]\left[\mathrm{PF}_{6}^{-}\right]$and aqueous glycerol were performed. Since the SED theory predicts a linear relationship between the solute reorientation time and solvent viscosity, $\tau_{r}$ vs $\eta / T$ for DODCI and MC 540 in the two solvents are plotted in figure 13. The SED lines with stick boundary condition are also displayed in the figure. The hydrodynamic volumes of the two solutes were obtained from the slopes of the plots of $\tau_{r}$ vs $\eta / T$ in $\left[\mathrm{bmim}^{+}\right]\left[\mathrm{PF}_{6}^{-}\right]$and aqueous glycerol. Such calculation reveals that the hydrodynamic volume of DODCI in aqueous glyc-
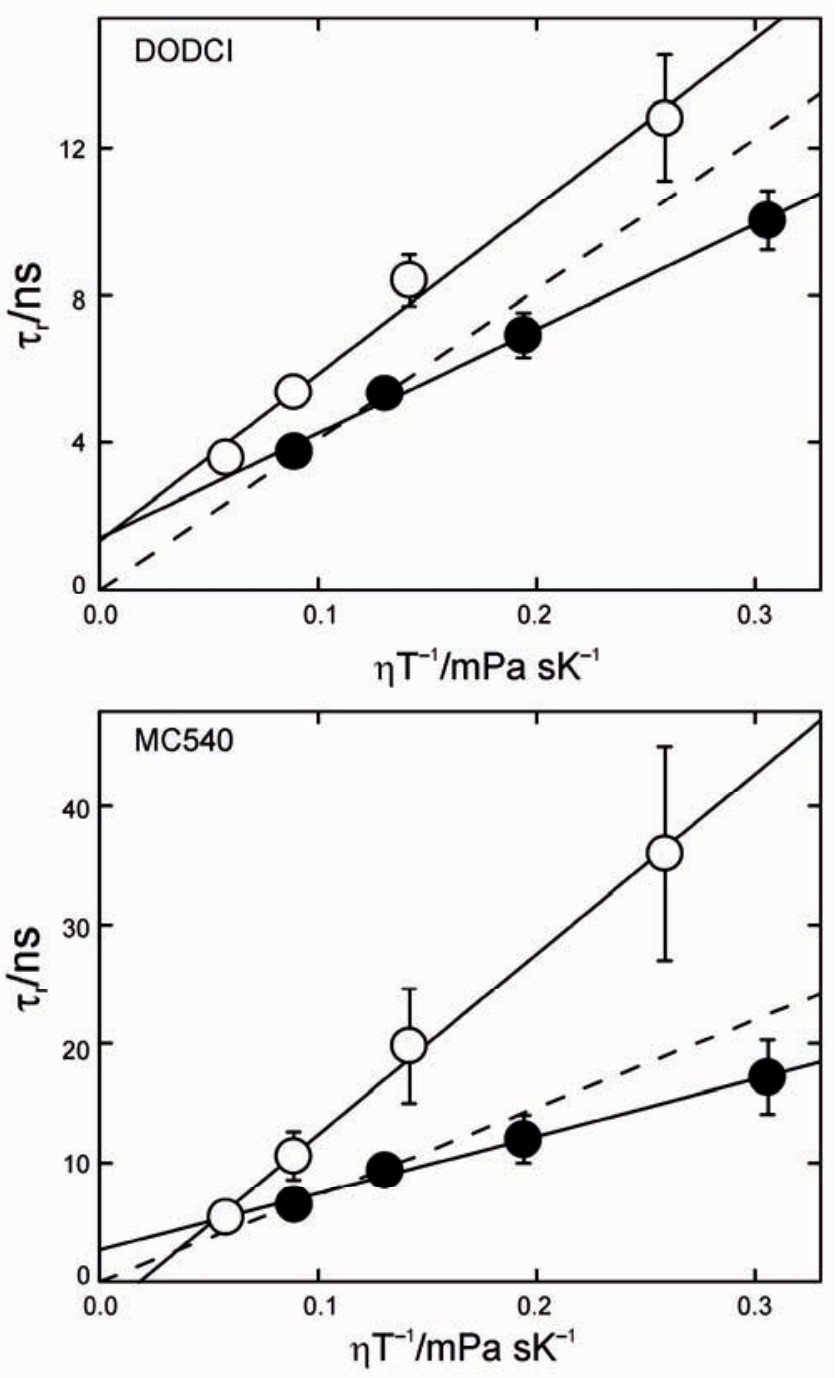

Figure 13. Plots of $\tau_{r}$ vs $\eta / T$ for DODCI and MC 540 in $\left[\mathrm{bmim}^{+}\right]\left[\mathrm{PF}_{6}^{-}\right](\bullet)$ and aqueous glycerol $(\mathrm{O})$. The lines passing through the data points were obtained by linear least-squares fit. The dashed lines are the calculated ones using SED theory with stick boundary condition. Reprinted with permission from ref 48. Copyright (2008) American Institute of Physics. erol is larger by a factor of 1.5 compared to that in $\left[\mathrm{bmim}^{+}\right]\left[\mathrm{PF}_{6}^{-}\right]$. It has been established that solvent attachment does not play an important role in the rotational diffusion of DODCI. ${ }^{96}$ Thus the observed slower rotation of DODCI in aqueous glycerol compared to $\left[\mathrm{bmim}^{+}\right]\left[\mathrm{PF}_{6}^{-}\right]$is a consequence of the smaller size of glycerol compared to the ionic liquid. It may be recalled that, in the absence of specific solutesolvent interactions, an increase in the solvent size reduces the friction experienced by the rotating solute molecule, leading to a faster rotation. This aspect has been experimentally verified in case of a number of non-polar solutes in non-polar and/or dipolar solvents ${ }^{52,59-65}$ and also in $\left[\mathrm{bmim}^{+}\right]\left[\mathrm{PF}_{6}^{-}\right] .{ }^{47} \mathrm{In}$ contrast to DODCI, the hydrodynamic volume of MC 540 obtained in aqueous glycerol is larger by over a factor of 3 compared to that in the ionic liquid. This observation indicates that specific solutesolvent interactions are playing a significant role in the rotational diffusion of MC 540. Thus, the rotational diffusion studies carried out in this manner provide evidence to the hypothesis that in case of MC 540 in aqueous glycerol, specific solute-solvent interactions are indeed stabilizing the zwitterionic structures of the solute, thereby lowering the barrier for isomerization. Apart from lowering the barrier height, specific solute-solvent interactions also increase the friction for rotational diffusion as well as twisting motion. It is a well-established fact that in the intermediate and high friction regimes, the rate constant for isomerization decreases as the friction increases. ${ }^{51,76}$ However, in case of MC 540 in aqueous glycerol, the effect of an increase in the friction on the photoisomerization rate constant is more than compensated by the lowering of the barrier height due to specific solute-solvent interactions.

\section{Summary and outlook}

Although the understanding of solute-solvent interactions in molecular liquids has come a long way, these aspects have received a little attention in case of ionic liquids. A thorough understanding of various aspects of solute-solvent interactions will bring considerable advances in our ability to probe, analyse, comprehend, and even predict the physicochemical properties of new generation of "tailor made' ionic liquids. In this article an attempt has been made to explore the role of solute-solvent interactions on two friction dependent processes namely rotational diffusion and photoisomerization 
in a typical ionic liquid. Following is the summary and outlook of the work presented in this short review.

The experience and expertise gleaned over the years in understanding solute rotation and photoisomerization in conventional liquids can be used to explore the nature of solute-solvent interactions in ionic liquids as well. Comparing the rotational diffusion of a pair of structurally similar non-dipolar solutes, DMDPP and DPP demonstrates that organic solutes do experience strong hydrogen bonding interactions with ionic liquids despite their highly associative nature. Similar studies have been carried out with structurally similar non-polar solute, 9PA and an ionic solute, R110 in $\left[\mathrm{bmim}^{+}\right]\left[\mathrm{PF}_{6}^{-}\right]$and glycerol to understand the interplay of size effects and specific interactions on rotational diffusion. A comparison of the results under isoviscous conditions reveals that the rotational diffusion of the non-polar solute is mainly governed by solvent-size and free volume. In contrast, for ionic solutes it is the solutesolvent interaction strength that dictates the rotational diffusion. Photoisomerization studies carried out with a pair of cyanine derivatives (DODCI and MC 540) in $\left[\mathrm{bmim}^{+}\right]\left[\mathrm{PF}_{6}^{-}\right]$, on the other hand, indicate that the rates of isomerization are influenced only by the viscosity of the medium.

Despite the insights gained from the efforts of various researchers, a comprehensive understanding of solute-solvent interactions in ionic liquids is still far from complete. Various aspects such as the effect of alkane side chains of the cations, charge to size ratio of the anions and overall dielectric properties of the ionic liquids on the dynamics of solutes dissolved in them need to be investigated systematically. Besides these issues, most (although not all) of the investigations carried out so far have dealt with imidazolium based ionic liquids. As a consequence, it is necessary to extend these studies to other types of ionic liquids because the number of newly synthesized ionic liquids is increasing at an appreciable rate. In essence, the studies presented in this short review, although provide a glimpse of understanding of some of the very intriguing aspects; they need to be extended to newer systems in order to get a comprehensive picture of solute-solvent interactions in ionic liquids.

\section{References}

1. Welton T 1999 Chem. Rev. 992071

2. Clean solvents: Alternative media for chemical reactions and processing (eds) M A Abraham and L
Moens 2002 (Washington, DC: American Chemical Society) and references therein

3. Adams D, Dyson P and Tavener S 2004 In Chemistry in alternative reaction media (New York: John Wiley \& Sons)

4. Welton T 2004 Coord. Chem. Rev. 2482459

5. Wasserscheid P and Keim W 2000 Angew. Chem., Int. Ed. 393773

6. Ionic liquids in synthesis 2002 (eds) P Wasserscheid and T Welton (VCH-Wiley, Weinheim)

7. Ionic liquids as green solvents: Progress and prospects 2003 (eds) R D Rogers and K R Seddon (Washington DC: American Chemical Society)

8. West A 2005 Chem. World 33

9. Swatloski R P, Holbrey J D and Rogers R D 2003 Green Chem. 5361

10. Abedin S Z E and Endres F 2007 Acc. Chem. Res. 40 1106

11. Antony J H, Mertens D, Dölle A, Wasserscheid P and Carper W R 2003 Chem. Phys. Chem. 4588

12. Fadeev A G and Meagher M M 2001 Chem. Commun. 295

13. Dzyuba S V and Bartsch R A 2002 Chem. Phys. Chem. 3161

14. Baker S N, Baker G A, Kane M A and Bright F V 2001 J. Phys. Chem. B105 9663

15. Ingram J A, Moog R S, Ito N, Biswas R and Maroncelli M 2003 J. Phys. Chem. B107 5926

16. Ito N, Arzhantsev S, Heitz M and Maroncelli M 2004 J. Phys. Chem. B108 5771

17. Ito N, Arzhantsev S and Maroncelli M 2004 Chem. Phys. Lett. 39683

18. Chakrabarty D, Chakraborty A, Seth D, Hazra P and Sarkar N 2004 Chem. Phys. Lett. 397469

19. Chakrabarty D, Chakraborty A, Seth D and N Sarkar 2005 J. Phys. Chem. A109 1764

20. Hanke C G, Price S L and Lynden-Bell R M 2001 Mol. Phys. 99801

21. Aki S N V K, Brennecke J F and Samanta A 2001 Chem. Commun. 413

22. Fletcher K A, Storey I A, Hendricks A E, Pandey S and Pandey S 2001 Green Chemistry 3210

23. Karmakar R and Samanta A 2002 J. Phys. Chem. A106 4447

24. Karmakar R and Samanta A 2002 J. Phys. Chem. A106 6670

25. Arzhantsev S, Ito N, Heitz M and Maroncelli M 2003 Chem. Phys. Lett. $\mathbf{3 8 1} 278$

26. Samanta A 2006 J. Phys. Chem. B110 13704

27. Arzhantsev S, Jin H, Baker G A and Maroncelli M 2007 J. Phys. Chem. B111 4978

28. Kashyap H K and Biswas R 2008 J. Phys. Chem. B12 12431

29. Chakrabarty D, Chakraborty A, Hazra P, Seth D and Sarkar N 2004 Chem. Phys. Lett. 397216

30. Paul A and Samanta A 2008 J. Phys. Chem. B112 947

31. Anderson J L, Pino V, Hagberg E C, Sheares V V, and Armstrong, D W 2003 Chem. Commun. 2444

32. Fletcher K A and Pandey, S 2004 Langmuir 2033

33. Gao H, Li J, Han B, Chen W, Zhang J, Zhang R and Yan D 2004 Phys. Chem. Chem Phys. 62914 
34. Gao Y, Han S, Han B, Li G, Shen D, Li Z, Du J, Hou W and Zhang G 2005 Langmuir 215681

35. Eastoe J, Gold S, Rogers S E, Paul A, Welton T, Heenan R K and Grillo I J $2005 \mathrm{~J}$. Am. Chem. Soc. 1277302

36. Patrascu C, Gauffre F, Nallet F, Bordes R, Oberdisse J, de Lauth-Viguerie N and Mingotaud C 2006 Chem. Phys. Chem. 799

37. Araos M U and Warr G G 2008 Langmuir 249354

38. Zhang S, Li N, Zheng L, Li X, Gao Y and Yu L 2008 J. Phys. Chem. B112 10228

39. Chakraborty A, Seth D, Chakrabarty D, Setua P and Sarkar N 2005 J. Phys. Chem. A109 11110

40. Seth D, Chakraborty A, Setua P and Sarkar N $2007 \mathrm{~J}$. Phys. Chem. B111 4781

41. Chakrabarty D, Seth D, Chakraborty A and Sarkar N 2005 J. Phys. Chem. B109 5753

42. Seth D, Chakraborty A, Setua P and Sarkar N 2006 Langmuir 227768

43. Ozawa R and Hamaguchi H 2001 Chem. Lett. 736

44. Lee C, Mamantov G and Pagni R M 2002 J. Chem. Res. 122

45. Shim T, Hee Lee M, Kim D and Ouchi Y $2008 \mathrm{~J}$. Phys. Chem. B112 1906

46. Mali K S, Dutt G B and Mukherjee T 2005 J. Chem. Phys. 123174504

47. Mali K S, Dutt G B and Mukherjee T 2008 J. Chem. Phys. 128054504

48. Mali K S, Dutt G B and Mukherjee T 2008 J. Chem. Phys. 128124515

49. Kivelson D 1987 In Rotational dynamics of small and macromolecules (eds) Th Dorfmüller and R Pecora (Berlin: Springer-Verlag)

50. Fleming G R 1986 In Chemical applications of ultrafast spectroscopy (New York: Oxford University Press) and references therein

51. Waldeck D H 2000 In Conformational analysis of molecules in the excited state (ed.) J Waluk (New York: Wiley-VCH:) p. 113 and references therein

52. Dutt G B 2005 Chem. Phys. Chem. 6413 and references therein

53. Einstein A 1956 In Investigations on the theory of Brownian movement (New York: Dover)

54. Dutt G B and Ghanty T K 2003 J. Chem. Phys. 119 4768

55. Perrin F 1934 J. Phys. Radium 5497

56. $\mathrm{Hu} \mathrm{C} \mathrm{M}$ and Zwanzig R 1974 J. Chem. Phys. 60 4354

57. Dutt G B, Srivatsavoy V J P and Sapre A V $1999 \mathrm{~J}$. Chem. Phys. 1109623

58. Ben-Amotz D and Scott T W 1987 J. Chem. Phys. 87 3739

59. Ben-Amotz D and Drake J. M 1988 J. Chem. Phys. 891019

60. De Backer S, Dutt G B, Ameloot M, De Schryver F C, Müllen K and Holtrup F 1996 J. Phys. Chem. 100512

61. Roy M and Doraiswamy S 1993 J. Chem. Phys. 98 3213

62. Anderton R M and Kauffman J F 1994 J. Phys. Chem. 9812117
63. Kim S K and Fleming G R 1988 J. Phys. Chem. 92 2168

64. Benzler J and Luther K 1997 Chem. Phys. Lett. 279333

65. Courtney S H, Kim S K, Canonica S and Fleming G R 1986 J. Chem. Soc. Faraday Trans. 2822065

66. Bowman R M, Eisenthal K B and Millar D P $1988 \mathrm{~J}$. Chem. Phys. 89762

67. Jiang Y and Blanchard G J 1994 J. Phys. Chem. 986436

68. Jas G S, Wang Y, Pauls S W, Johnson C K and Kuczera K 1997 J. Chem. Phys. 1078800

69. Brocklehurst B and Young R N 1995 J. Phys. Chem. 9940

70. Adachi M and Nakamura S 1994 J. Phys. Chem. 981796

71. Gierer A and Wirtz K 1953 Naturforsch. A8 532

72. Dote J L, Kivelson D and Schwartz R N 1981 J. Phys. Chem. 852169

73. Chuang T J and Eisenthal K B 1971 Chem. Phys. Lett. 11368

74. Eisenthal K B 1975 Acc. Chem. Res. 8118

75. Dutt G B and Ghanty T K 2004 J. Phys. Chem. A108 6090

76. Waldeck D H 1991 Chem. Rev. 91415 and references therein

77. Velsko S P and Fleming G R 1982 J. Chem. Phys. 76 3553

78. Keery K M and Fleming G R 1982 Chem. Phys. Lett. 93322

79. Lee M, Bain A J, McCarthy P J, Han C H, Haseltine J N, Smith III A B and Hochstrasser R M $1986 J$. Chem. Phys. 854341

80. Lee M, Haseltine J N, Smith III A B and Hochstrasser R M $1989 \mathrm{~J}$. Am. Chem. Soc. 1115044

81. Anderton R M and Kauffman J F 1994 J. Phys. Chem. 9812125

82. Anderton R M and Kauffman J F 1995 J. Phys. Chem. 9914628

83. Rullière C 1976 Chem. Phys. Lett. 43303

84. Sundström V and Gillbro T 1982 J. Phys. Chem. 86 1788

85. Velsko S P and Fleming G R 1982 Chem. Phys. 6559

86. Velsko S P, Waldeck D H and Fleming G R $1983 \mathrm{~J}$. Chem. Phys. 78249

87. Hara K and Akimoto S 1991 J. Phys. Chem. 955811

88. Hoebeke M, Piette J and Vorst A V $1990 \mathrm{~J}$. Photochem. Photobiol. B. Biol. 4273

89. Aramendía P F, Kreig M, Nitsch C, Bittersmann E and Braslavsky S E 1988 Photochem. Photobiol. 48187

90. Aramendía P F, Duchowicz R, Scaffardi L and Tocho J O 1990 J. Phys. Chem. 941389

91. Onganer Y, Yin M, Bessire D R and Quitevis E L 1993 J. Phys. Chem. 972344

92. Aramendía P F, Negri R M and Román E S $1994 \mathrm{~J}$. Phys. Chem. 983165

93. Kramers H A 1940 Physica 7284

94. Lackowicz J R 1983 Principles of fluorescence spectroscopy (Plenum: New York)

95. Paul A, Mandal P K and Samanta A 2005 Chem. Phys. Lett. 402375

96. Fleming G R, Knight A E W, Morris J M, Robbins R J and Robinson G W 1977 Chem. Phys. Lett. 491 